\title{
CARDIAC IMAGING IN CORONARY ARTERY DISEASE: DIFFERING MODALITIES
}

\section{J D Schuijf, L J Shaw, W Wijns, H J Lamb, D Poldermans, A de Roos, E E van der Wall, J J Bax}

Heart 2005;91:1110-1117. doi: 10.1136/hrt.2005.061408

Take the online multiple choice questions associated with this article (see page 1104)
See end of article for authors' affiliations

Correspondence to: Dr Jeroen J Bax, Department of Cardiology, Leiden University Medical Center, Albinusdreef 2, 2333 ZA Leiden, The Netherlands; jbax@knoware.nl

\footnotetext{
C
} oronary artery disease (CAD) remains one of the leading causes of morbidity and mortality worldwide. Moreover, the disease is reaching endemic proportions and will put an enormous strain on health care economics in the near future. Non-invasive testing is important to exclude CAD with a high certainty on the one hand, and to detect CAD with its functional consequences at an early stage, to guide optimal patient management, on the other hand. For these purposes, non-invasive imaging techniques have been developed and used extensively over the last years. Currently, the main focus of non-invasive imaging for diagnosis of CAD is twofold: (1) functional imaging, assessing the haemodynamic consequences of obstructive coronary artery disease; and (2) anatomical imaging, visualising non-invasively the coronary artery tree.

For functional imaging, nuclear cardiology, stress echocardiography, and magnetic resonance imaging (MRI) are used, whereas for anatomical imaging or non-invasive angiography, MRI, multislice CT (MSCT), and electron beam CT (EBCT) are used.

This manuscript will update the reader on the current status of non-invasive imaging, with a special focus on functional imaging versus anatomical imaging for the detection of CAD. The accuracies of the different imaging modalities are illustrated using pooled analyses of the available literature data when available.

\section{FUNCTIONAL IMAGING}

What information does functional imaging provide?

The hallmark of functional imaging is the detection of CAD by assessing the haemodynamic consequences of CAD rather than by direct visualisation of the coronary arteries. For this purpose, regional perfusion or wall motion abnormalities are induced (or worsened) during stress, reflecting the presence of stress induced ischaemia. Ischaemia induction is based on the principle that although resting myocardial blood flow in regions supplied by stenotic coronary arteries is preserved, the increased flow demand during stress cannot be met, resulting in a sequence of events referred to as "the ischaemic cascade". ${ }^{1}$ Initially perfusion abnormalities are induced, followed by diastolic and (at a later stage) systolic dysfunction; only at the very end of the cascade do ECG changes and angina occur (fig 1). Accordingly, the occurrence of perfusion abnormalities during stress may be more sensitive for the detection of CAD than the induction of systolic dysfunction (wall motion abnormalities).

Currently, functional imaging can be performed using (gated) single photon emission computed tomography (SPECT) or positron emission tomography (PET), (contrast) stress echocardiography, and MRI; all techniques allow integrated assessment of perfusion and function, at rest and after stress, and are used clinically according to local availability and expertise.

\section{Types of stress}

An increased demand can be achieved through physical (bicycle or treadmill) exercise, or (in patients unable to exercise) pharmacological stress can be applied including adrenergic stimulation and vasodilation. Dobutamine (a $\beta 1$ specific agonist) increases heart rate, contractility, and arterial blood pressure, resulting in increased myocardial oxygen demand. Dobutamine is administered intravenously at incremental doses of 5, 10, 20, 30, and $40 \mu \mathrm{g} / \mathrm{kg} / \mathrm{min}$ at intervals of approximately five minutes. When the target heart rate is not reached, atropine $(0.25-1.0 \mathrm{mg})$ can be added; $\beta$ blockers can be used as antidote.

The vasodilators include dipyridamole and adenosine. Adenosine is a direct vasodilator, while dipyridamole inhibits cellular uptake and breakdown of adenosine. Dipyridamole therefore has a slower onset, while its effect lasts longer. For adenosine, a stepwise infusion protocol can be used, consisting of three-minute stages of $0.10,0.14$, and $0.18 \mathrm{mg} / \mathrm{kg} / \mathrm{min}$. Dipyridamole is 


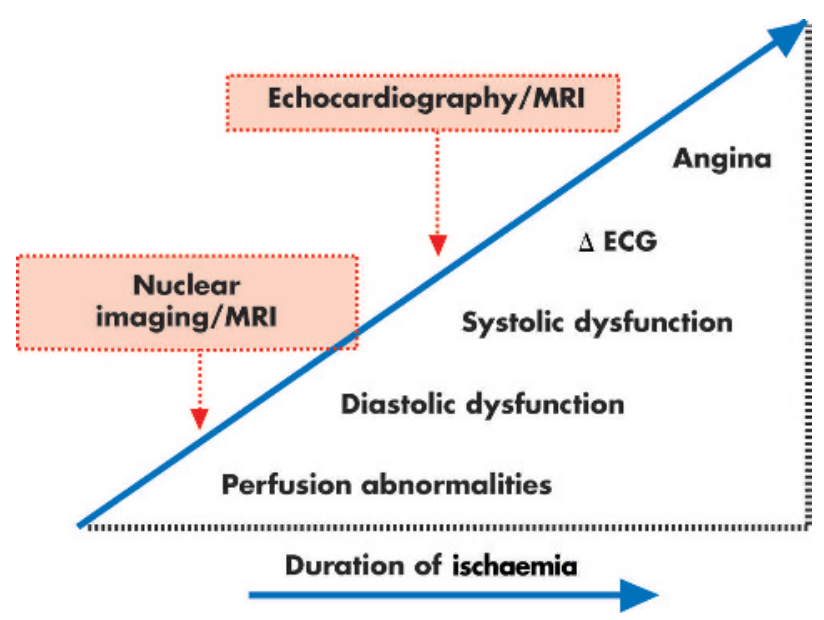

Figure 1 The ischaemic cascade represents the sequence of pathophysiological events following ischaemia.

administered intravenously over four minutes (dose $0.56 \mathrm{mg}$ / $\mathrm{kg}$ ), followed by a second dose of $0.28 \mathrm{mg} / \mathrm{kg}$. Aminophylline can be used as antidote.

Safety of all pharmacological stressors has been investigated extensively and, although continuous patient monitoring is required, severe complications are rare. ${ }^{23}$

\section{Which modalities are available for functional imaging? \\ SPECT: assessment of perfusion}

Most experience for assessment of perfusion in daily clinical practice has been obtained with SPECT. Three radiopharmaceuticals are used: thallium-201, technetium-99m sestamibi, and technetium-99m tetrofosmin. Currently, the technetium$99 \mathrm{~m}$ labelled tracers are preferred for their higher photon energy resulting in less attenuation artefacts. Two sets of images are obtained: after stress and at rest. In general, reversible and irreversible defects are considered indicative of CAD. While reversible (stress induced) defects reflect ischaemia, irreversible (fixed) defects mainly represent infarcted myocardium (fig 2). Images are interpreted visually or using automated quantification. For segmentation of the left ventricle (LV), a 17 segment model is developed, that can be applied to all functional imaging modalities (fig 3). ${ }^{4}$ To assess the diagnostic accuracy of SPECT for detection of CAD, Underwood et al pooled 79 studies ( $\mathrm{n}=8964$ patients) showing a weighted mean sensitivity and specificity of $86 \%$ and $74 \%^{5}$ (fig 4). The lower specificity of SPECT may be (partially) attributable to referral bias-that is, among

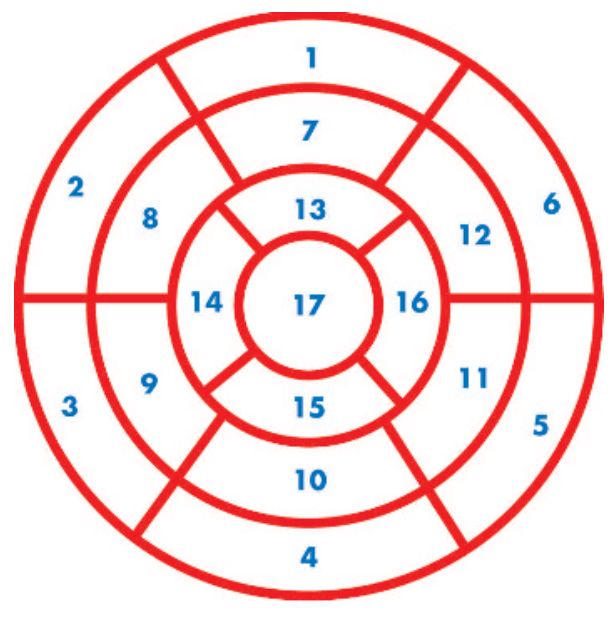

1111

Figure 3 Schematic presentation of the 17 segment model (adapted from Cerqueira et $\left.a^{4}\right)$. Besides its application for evaluation of SPECT images, this model can be applied to echocardiography and MRI as well.

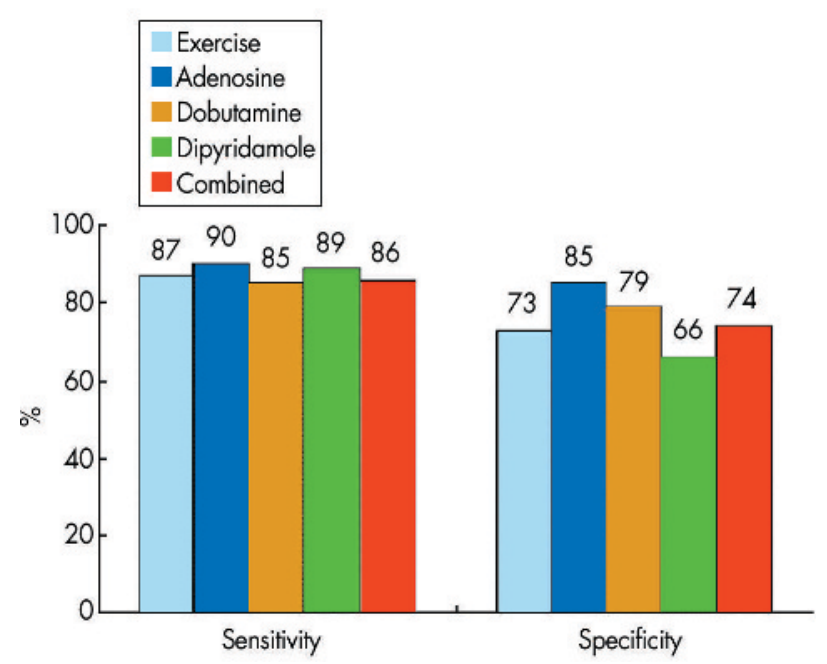

Figure 4 Sensitivities and specificities of SPECT imaging for the detection of coronary artery disease, using different stressors (data are based on Underwood et $a^{5}$ ).

patients with normal SPECT studies, only those with a high suspicion for CAD are referred for coronary angiography. To overcome this problem, the normalcy rate has been introduced, which is the percentage of normal SPECT studies in a

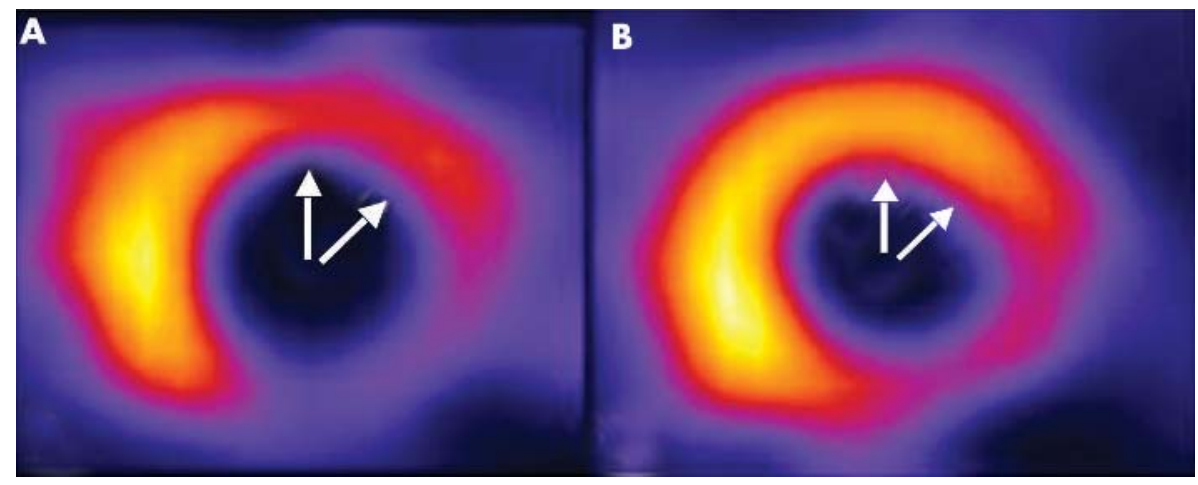

Figure 2 Example of a reversible defect on technetium- $99 \mathrm{~m}$ tetrofosmin SPECT. Panels A and B show short axis slices following stress and at rest, respectively. A reversible defect is present in the anterior and anterolateral regions (white arrows), illustrating stress inducible ischaemia. A fixed perfusion defect, most likely representing scar tissue, is present in the posterolateral and inferior region. 
population with a low likelihood of CAD. Pooled analysis of 10 studies ( $\mathrm{n}=543$ patients) showed a normalcy rate of $89 \%{ }^{5}$

\section{SPECT: assessment of systolic function}

The introduction of ECG gated SPECT imaging has allowed assessment of global and regional LV function in addition to perfusion. Direct comparisons between gated SPECT and MRI (or echocardiography) showed excellent correlations for assessment of LV ejection fraction, volumes, and regional wall motion. ${ }^{67}$ Addition of these systolic function parameters has improved diagnostic accuracy. In particular, artefacts caused by soft tissue attenuation could be unmasked by the demonstration of normal wall motion. This resulted in a substantial reduction of false positive test results. ${ }^{8}$ Integration of perfusion and systolic function by SPECT resulted in a significant reduction (from $31 \%$ to $10 \%$ ) of inconclusive tests, with an increase in normalcy rate from $74 \%$ to $93 \%{ }^{9}$

Echocardiography: assessment of systolic function Stress echocardiography is readily available for the routine evaluation of (stress inducible) wall motion abnormalities (fig 5); both resting and stress induced (or worsened) wall motion abnormalities are indicative of CAD. While stress induced (or worsened) wall motion abnormalities reflect ischaemia, resting wall motion abnormalities mainly represent infarcted myocardium. A total of 15 studies $(\mathrm{n}=1849$ patients) used exercise echocardiography to detect CAD, with a weighted mean sensitivity and specificity of $84 \%$ and $82 \%{ }^{10}$ Pooled data from 28 dobutamine echocardiography studies ( $\mathrm{n}=2246$ patients) showed a weighted mean sensitivity and specificity of $80 \%$ and $84 \%$ to detect CAD. ${ }^{10}$ The accuracies for the different forms of stress echocardiography are summarised in fig 6 . It has been demonstrated that the continuation of $\beta$ blockers reduced sensitivity, which could be improved by addition of atropine. Also, sensitivity increased in parallel to the number of diseased vessels, from $74 \%$ for one vessel disease to $92 \%$ for three vessel disease. Disadvantages of stress echocardiography in general include a suboptimal acoustic window in up to $25 \%$ of patients and dropout of the anterior and lateral walls. Improved endocardial border delineation can be obtained by using second harmonic imaging and administration of intravenous contrast agents.

\section{Echocardiography: assessment of perfusion}

At the same time the use of contrast agents has allowed the assessment of myocardial perfusion. After contrast injection, the microbubbles remain in the vascular space until they dissolve, and thus reflect the microvascular circulation. Accordingly, their relative concentrations in different regions of the myocardium (as measured by signal intensity) reflect the relative myocardial blood volume in those regions. Similar to SPECT, resting perfusion defects suggest infarcted myocardium, whereas stress induced perfusion defects indicate ischaemia (fig 7). Currently, many modifications of

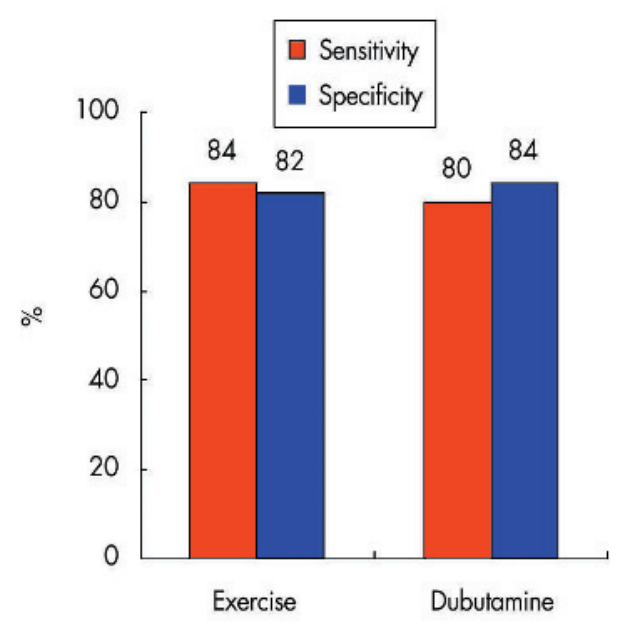

Figure 6 Diagnostic accuracy of stress (exercise and dobutamine) echocardiography (data based on Bax et a ${ }^{10}$ ).
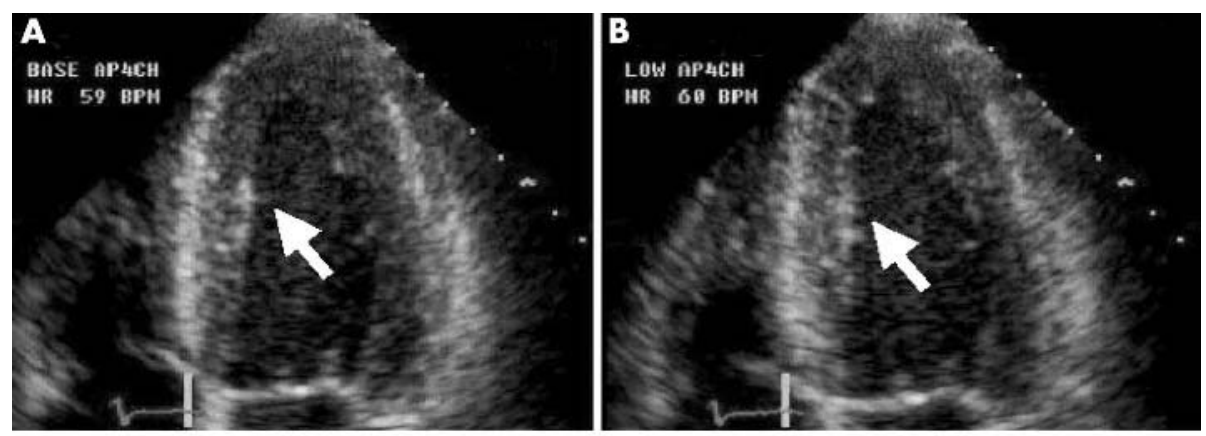

Figure 5 Example of a stress induced wall motion abnormality on dobutamine echocardiography. Panels A, B, C, and $D$ are obtained during rest, low dose $(10 \mu \mathrm{g} / \mathrm{kg} / \mathrm{min})$ and high dose dobutamine $(40 \mu \mathrm{g} / \mathrm{kg} / \mathrm{min})$, and recovery. In the septal region (white arrow), normal wall motion is present at rest and during low dose dobutamine infusion, whereas dyskinesia is induced at high dose dobutamine.
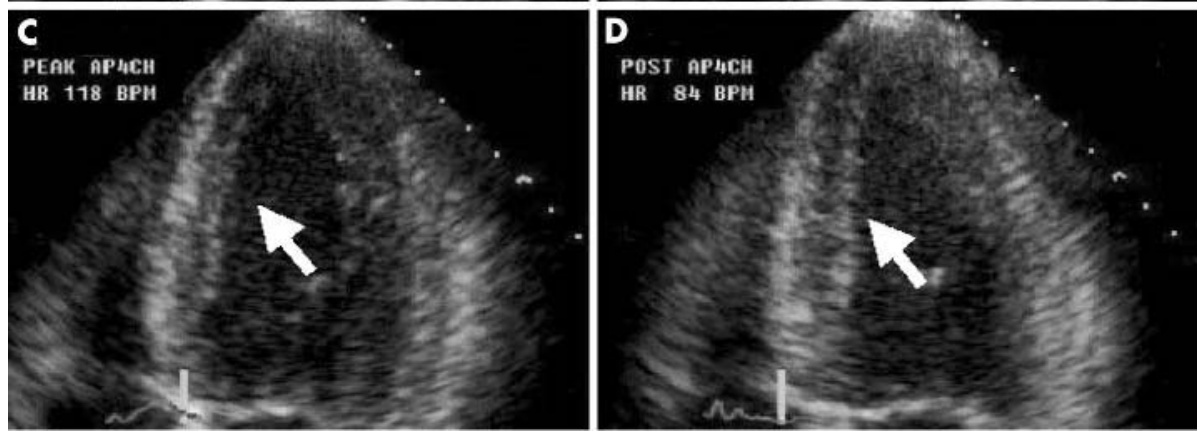

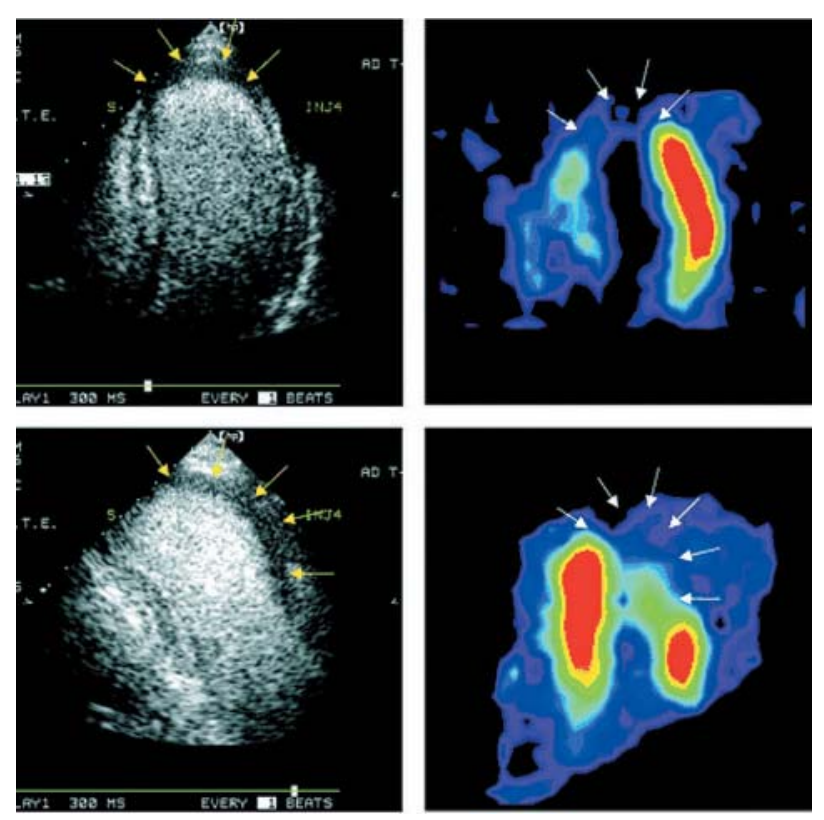

Figure 7 Representative myocardial contrast echocardiography (MCE) and SPECT images obtained in a patient with anterior myocardial infarction, showing a larger perfusion defect by SPECT than MCE: (top left) apical four chamber MCE; (bottom left) apical two chamber MCE; (top right) apical four chamber SPECT; (bottom right) apical two chamber SPECT. Arrows delineate the area of decreased opacification/tracer uptake. Reprinted from Jucquois et al ${ }_{1}^{14}$ with permission of BMJ Publishing Group Ltd.

the technology have been introduced and real time assessment of perfusion by contrast echocardiography is now possible. ${ }^{11}$

Recent studies from experienced centres showed an excellent agreement between SPECT and myocardial contrast echocardiography for detection of perfusion abnormalities, with a comparable sensitivity/specificity for the detection of CAD. ${ }^{12}{ }^{13}$ In a head-to-head comparison, Jucquois et $a l^{14}$ demonstrated an agreement of $62 \%$ between SPECT and contrast echocardiography for detection of perfusion defects; the disagreement between the two techniques was related to attenuation artefacts and when these segments were excluded, the concordance improved to $82 \%$.

The integration of assessment of perfusion and function by contrast echocardiography performed at rest and after stress should provide optimal information on the detection of CAD. Moir et al recently performed myocardial contrast echocardiography in addition to combined dipyridamole exercise echocardiography in 85 patients. ${ }^{15}$ In 70 of these patients, data could be compared to conventional coronary angiography. Sensitivity for the detection of CAD was significantly improved by the addition of contrast from $74 \%$ to $91 \%$; specificity on the other hand showed a (non-significant) decrease from $81 \%$ to $70 \%$. Pooled analysis of the seven currently available studies ( $\mathrm{n}=245$ patients) on the additive value of perfusion imaging with contrast to standard wall motion imaging showed similar results: the weighted mean sensitivity for detection of CAD was $89 \%$ with a specificity of $63 \% .^{15-21}$

\section{MRI: assessment of perfusion}

A relatively new technique to evaluate myocardial perfusion is MRI. For this purpose, 5-8 slices in the short axis

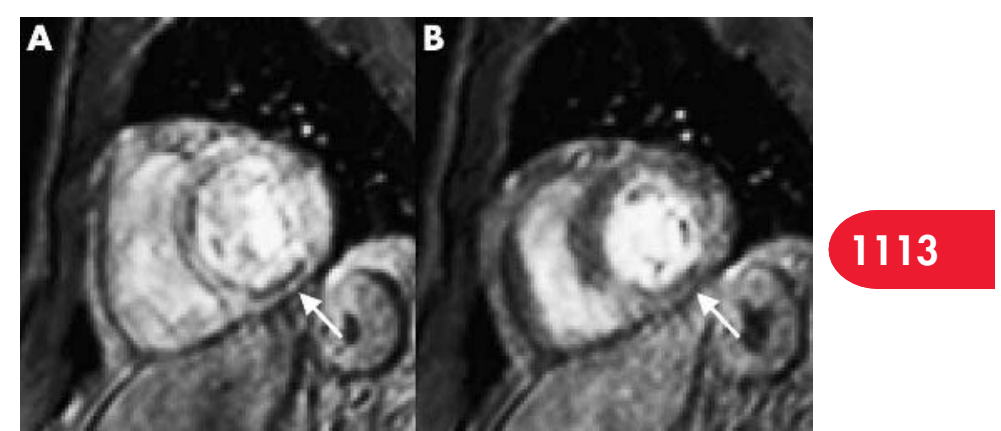

Figure 8 Magnetic resonance perfusion images during rest (panel A) and stress (panel B) showing a fixed perfusion defect in the inferior wall (white arrows). Images were acquired using a breath hold sensitivity encoding imaging technique during the first pass of an intravenously administered bolus of gadolinium contrast agent.

orientation are imaged during the first pass of a bolus of a contrast agent. Imaging is repeated during pharmacological stress. The applied contrast agent, gadolinium, temporarily changes the $\mathrm{Tl}$ relaxation time and thereby increases the signal intensity of the perfused myocardium. In contrast, ischaemic regions are identified as areas with little or reduced signal intensity (fig 8). Pooling of 17 MRI perfusion studies $(\mathrm{n}=502$ patients, using either dipyridamole or adenosine stress) revealed a weighted mean sensitivity and specificity of $84 \%$ and $85 \%$ (fig 9). ${ }^{1022-24}$ The high spatial resolution (approximately $2 \mathrm{~mm}$ ) enables distinction between subendocardial and transmural perfusion defects. This is an important advantage over SPECT imaging, since the occurrence of subendocardial perfusion defects may indicate compromised blood flow at an early stage.

For clinical routine, images are evaluated visually, although semi-quantitative assessment is possible by calculation of the myocardial perfusion reserve index..$^{25}$ In the future, absolute quantification of myocardial perfusion may be allowed by the use of new intravascular contrast agents. At present, however, quantitative analysis is still time consuming and in order to fully exploit this modality in

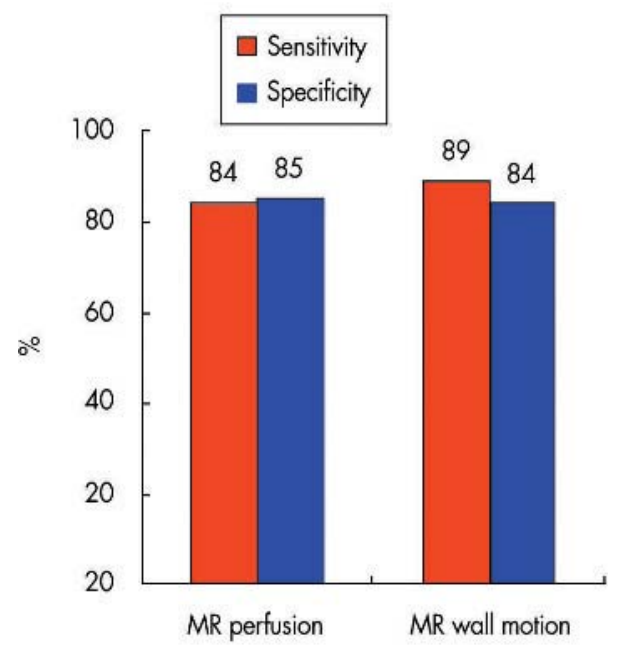

Figure 9 Diagnostic accuracy of perfusion and wall motion imaging MRI (data are based on several studies ${ }^{1022-24}$ ). For the perfusion studies, adenosine or dipyridamole were used, while dobutamine was administered during the wall motion studies. 
standard clinical routine, automated quantification algorithms are needed.

\section{MRI: assessment of systolic function}

In addition to myocardial perfusion, global and regional systolic LV function can also be obtained with MRI. The most

widely used steady state free precession technique allows clear identification of endocardial borders caused by a high blood pool signal. In addition, the tomographic approach allows measurement of volumes without geometric assumptions, resulting in accurate measurements in severely distorted ventricles as well. Global and regional LV function can be obtained at rest and during stress (mainly using dobutamine). Pooled data of 10 dobutamine MRI studies $(\mathrm{n}=654$ patients) revealed a weighted mean sensitivity and specificity of $89 \%$ and $84 \%$ (fig 9). ${ }^{1022}$

The excellent endocardial blood pool contrast is particularly beneficial for patients with poor echocardiographic windows. Unfortunately, MRI is still limited to highly specialised centres and acquisition protocols are still time consuming, making the technique currently unsuitable for evaluation of larger populations. No MRI studies with integration of systolic wall motion and perfusion to detect CAD are currently available.

\section{ANATOMICAL IMAGING \\ Why is anatomical imaging needed?}

Although a safe and accurate evaluation of patients with known or suspected CAD is offered by functional imaging, in a substantial number of patients anatomical imaging is needed. First, in patients with abnormal stress tests, direct visualisation of the coronary tree is still required for the definite diagnosis of CAD. Moreover, decisions on treatment strategy-for example, whether the observed coronary lesions will be treated conservatively (medically) or more aggressively by means of percutaneous coronary intervention (PCI) or coronary artery bypass graft surgery (CABG) - are based to a large extent on the findings of conventional coronary angiography. Also, in certain subpopulations-for example, diabetes-functional imaging may be less reliable. In these patients, diffuse atherosclerosis in all major epicardial vessels is frequently present, resulting in the absence of detectable perfusion abnormalities. Considering the fact that if CAD is present, prognosis is substantially worse compared to non-diabetic individuals, knowledge of coronary anatomy is needed. Thus, besides detection of haemodynamic consequences, direct visualisation of the coronary anatomy is frequently needed.

\section{What is the current gold standard for anatomical imaging?}

At present, conventional $x$ ray angiography with selective contrast injection through cardiac catheterisation remains the reference standard for the evaluation of the coronary arteries. Both spatial $(0.2 \mathrm{~mm})$ and temporal resolution ( $5 \mathrm{~ms}$ ) of the technique are extremely high. In addition, the degree of luminal narrowing can be precisely measured using quantitative coronary angiography. Also, when during the diagnostic procedure the presence of one or more significant lesions is confirmed, direct intervention is possible.

Currently, approximately 3000 invasive diagnostic procedures per million inhabitants have been performed in Europe in 2001, which resulted in percutaneous transluminal coronary angioplasty (PTCA) in only one out of three. ${ }^{27}$ The development of non-invasive imaging of the coronary arteries would potentially facilitate the access to anatomical imaging and expand the indications for revascularisation.

\section{What are the available modalities for non-invasive anatomical imaging?}

Currently, three techniques are being developed for noninvasive angiography: MRI, MSCT, and EBCT. Although results are promising, all techniques still have shortcomings and limitations, hampering implementation in routine clinical practice. Since the coronary arteries are small, tortuous, and show rapid movement during the cardiac cycle, demands on spatial and temporal resolution of the techniques are tremendous. However, all techniques are developing at a rapid pace and, as a result, image quality and diagnostic accuracy are continuously improving.

\section{Non-invasive angiography with MRI}

More than 10 years ago, the first results of non-invasive angiography were reported by Manning and colleagues. ${ }^{28}$ The authors performed a comparison between two dimensional MRI and conventional angiography in 39 patients and observed a sensitivity and specificity of $90 \%$ and $92 \%$, respectively. With these first generation techniques, data were acquired during consecutive breath holds, requiring substantial patient cooperation. To enable free breathing, navigator techniques, that allow real time monitoring of diaphragm motion, have been developed. In combination with the development of three dimensional acquisition techniques, superior visualisation of coronary anatomy was achieved. In fig 10, examples of non-invasive coronary angiography with three dimensional MR acquisition techniques are provided. Pooled data from 28 studies $(\mathrm{n}=903$ patients) directly comparing MRI with invasive angiography showed a weighted mean sensitivity of $72 \%$ with a specificity of $87 \%$ (fig 11). ${ }^{29}$ However, the percentage of interpretable segments is still insufficient and exclusion of up to $30 \%$ of all segments has been reported, even with newer acquisition techniques. Thus, full coverage of the coronary arteries within a reasonable amount of time still cannot be achieved. Future developments in the area of coronary MR angiography, including higher field strengths (3T) and improved contrast techniques, such as balanced steady state free precession techniques and the development of blood pool

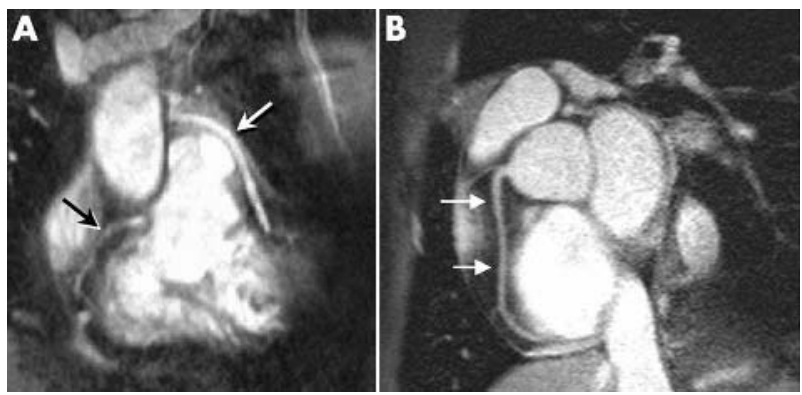

Figure 10 Non-invasive coronary angiography with MRI. In panel A a native right coronary artery (black arrow) and a venous coronary bypass (white arrow) on the left anterior descending coronary artery can be observed. In contrast, panel B depicts the right coronary artery (white arrows) of a healthy volunteer. Images were acquired with a $1.5 \mathrm{~T}$ system, using $\mathrm{T}_{2}$ preparation for background suppression during respiratory gating. 


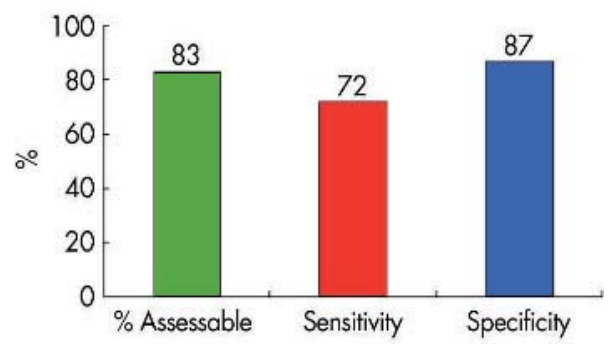

Figure 11 Diagnostic accuracy of non-invasive coronary angiography with MRI in the detection of significant stenoses (data based on Schuijf et $a^{29}$ ).

contrast agents, will improve diagnostic accuracy. Moreover, extensive research is directed towards assessment of plaque composition as well as assessment of coronary flow, which may potentially enable the technique to provide a comprehensive evaluation of both the presence and extent, as well as the functional significance, of CAD.

\section{Non-invasive angiography with MSCT}

More recently, MSCT has emerged as a potential modality for non-invasive angiography. Initial studies with four slice technology showed promising results, with sensitivities and specificities ranging from $66-90 \%$ and from $71-99 \%$, respectively. ${ }^{29}$ However, the technique was still hampered by the high percentage of segments (approximately 25\%) with nondiagnostic quality. Modern systems have an $x$ ray gantry rotation time of $400 \mathrm{~ms}$ or less while data are acquired using 16 or more parallel detectors with submillimetre collimation (fig 12). At present, 11 studies with 16 slice technology have been reported. ${ }^{29}$ As expected, considerably more segments were available for evaluation, approximately $96 \%$ of segments. Furthermore, an increase in sensitivity from (on average) $80 \%$ to $88 \%$ could also be observed with no loss in sensitivity (fig 13). With 64 slice systems that have recently become available, both the percentage evaluable segments and sensitivity are expected to improve further.

Since data are acquired during consecutive heartbeats, a stable heart rate is important in order to obtain good image quality. Similar to MRI, the technique has therefore limited value in patients with atrial fibrillation or frequent extrasystolic contractions, although for the latter raw data can sometimes be manually corrected. Other contraindications to MSCT include renal failure or pregnancy due to the administration of contrast agent and the use of ionising radiation, respectively. Moreover, the radiation dose associated with an MSCT examination is still considerably high and remains an important limitation of the technique. To reduce radiation dose, prospective $x$ ray tube modulation or more dedicated filtering may be applied while other dose reduction strategies are currently investigated.

\section{Non-invasive angiography with $\mathrm{EBCT}$}

The first experiences with coronary angiography with EBCT were described in $1995 .{ }^{30}$ Instead of a mechanically rotating tube, $x$ rays are created through an electron beam that is guided along a $210^{\circ}$ tungsten target ring in the gantry. As a result, a high resolution image is acquired in 50-100 ms. The acquisition of serial overlapping cross sectional images with a 1.5 or $3.0 \mathrm{~mm}$ slice thickness is performed during the administration of an iodinated contrast agent, using

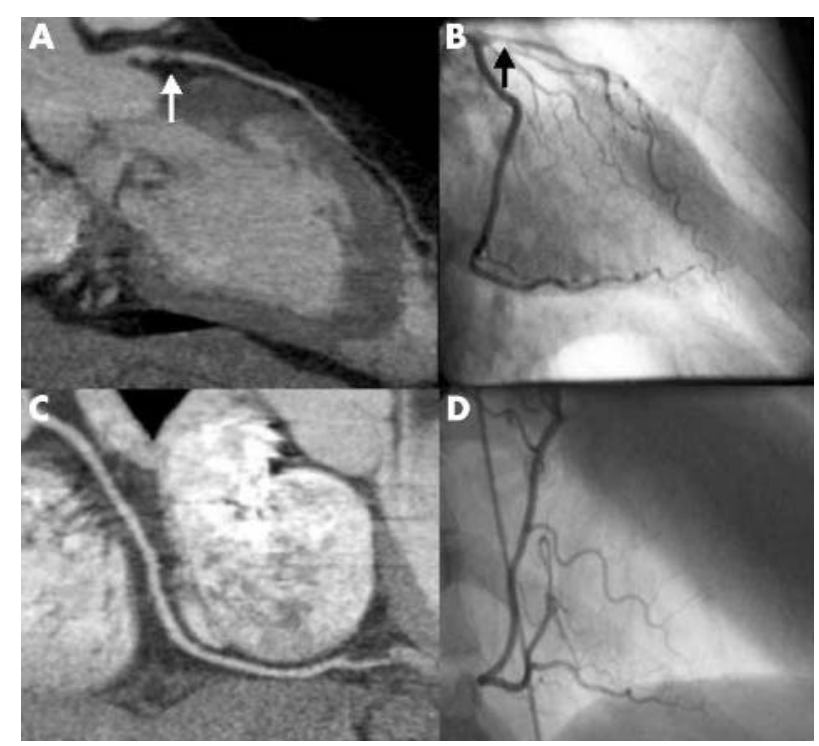

Figure 12 Non-invasive coronary angiography with 16 slice MSCT. In panel A, a curved multiplanar reconstruction of a left anterior descending coronary artery is depicted. A proximal stenosis can be observed (white arrow), which was confirmed by invasive angiography (panel $B$, black arrow). An example of a patent right coronary artery is provided in panel $C$. In panel $D$, the corresponding coronary angiogram is shown.

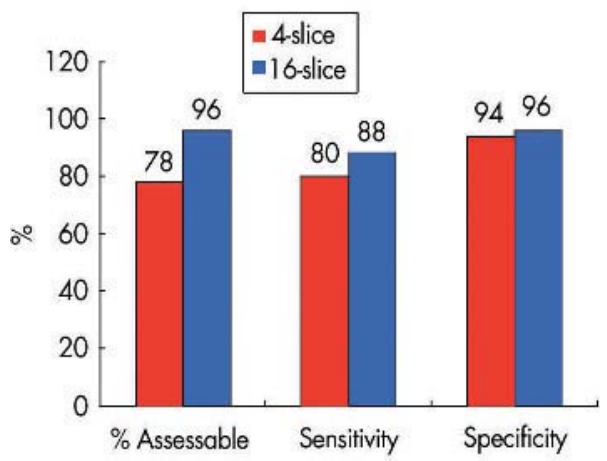

Figure 13 Diagnostic accuracy of non-invasive coronary angiography with four and 16 slice MSCT in the detection of significant stenoses (data based on Schuijf et $a^{p 9}$ ).

prospective ECG triggering. To cover the whole heart, 40-50 slices are necessary, typically requiring a breath hold of 30-40 seconds, depending on the heart rate. Pooled analysis of the 10 available studies ( $\mathrm{n}=583$ patients) comparing contrast enhanced EBCT angiography with conventional angiography demonstrated a weighted mean sensitivity and specificity of $87 \%$ and $91 \%$, respectively ${ }^{31} ; 16 \%$ of the coronary arteries were non-interpretable (fig 14). Similar to other non-invasive coronary angiography techniques, distal coronary segments are relatively more difficult to image, while coronary artery motion and breathing artefacts also frequently occur.

\section{Coronary artery calcium scoring}

Another, more frequently performed application of EBCT is the quantification of calcium in the coronary arteries. The presence of calcium serves as a marker of atherosclerosis. The absence of calcium virtually excludes atherosclerosis, and no further analysis is needed. This is also supported by the very 


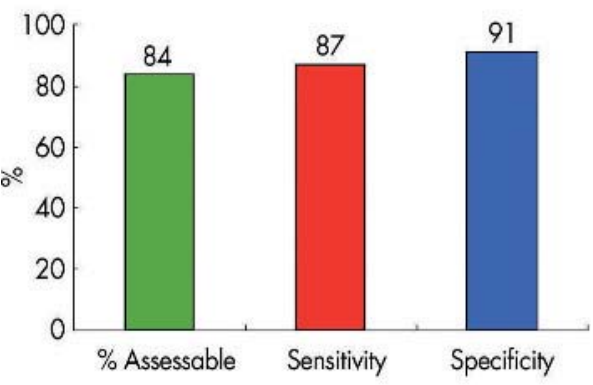

Figure 14 Image quality and diagnostic accuracy of EBCT (data based on Budoff et $a^{\beta 1}$ ).

low rate of cardiac events in patients without calcium on EBCT; Raggi et al ${ }^{32}$ demonstrated in 4800 patients without diabetes and no coronary calcium that the five year survival was $99.4 \%$. By multivariate analysis, the presence of coronary calcium contributed to the prediction of all cause mortality in 9474 asymptomatic and non-diabetic subjects to the same extent as age, hyperlipidaemia, hypertension, and active smoking. Moreover, Berman et $a l^{33}$ showed that $<1 \%$ of patients with minimal coronary calcium had ischaemia on SPECT imaging.

However, the presence of coronary calcium only indicates atherosclerosis in general and requires additional evaluation. In particular, no relation between the extent of coronary calcium and stenosis severity has been shown. ${ }^{31}$

\section{CONCLUSION}

As discussed in this manuscript, the emphasis of noninvasive imaging is on functional imaging (assessing the haemodynamic consequences of obstructive CAD-that is,

Cardiac imaging in coronary artery disease: key points

- In the presence of a significant coronary artery stenosis, a sequence of events called the "ischaemic cascade" occurs during stress: first perfusion abnormalities occur, followed by wall motion abnormalities, while ECG changes and angina occur at a later stage

- Non-invasive imaging to assess coronary artery disease can be divided into functional imaging and anatomical imaging

- Functional imaging aims at assessment of the haemodynamic consequences of obstructive coronary artery disease; the available techniques are nuclear imaging (mainly SPECT), stress echocardiography (with the optional use of intravenous contrast agents), and magnetic resonance imaging (MRI)

- Currently, all three functional imaging modalities allow comprehensive evaluation including assessment of both perfusion and wall motion

- For non-invasive anatomical imaging, or non-invasive coronary angiography, MRI, multislice CT (MSCT) and electron beam CT (EBCT) are used. These modalities do not yet assess the haemodynamic consequences of coronary artery disease

- In the future, integrated anatomical and functional imaging may eventually result in superior detection and evaluation of patients with suspected and known coronary artery disease ischaemia) and anatomic imaging (non-invasive angiography).

Over the past decades, non-invasive imaging for the detection of CAD has mainly relied on SPECT and stress echocardiography, functional imaging techniques to assess perfusion or wall motion abnormalities (as markers of CAD), respectively. Over time, these techniques were considered complementary, rather than competitive, since they provided different information. At present, however, both SPECT and echocardiography have developed into comprehensive imaging techniques, and each can assess both perfusion and wall motion. Similarly, MRI can also assess both perfusion and wall motion. Still, for proper patient management, knowledge on coronary anatomy is frequently needed and patients are subsequently referred for invasive angiography.

More recently, emphasis has shifted to anatomic imaging using MRI, MSCT, and EBCT allowing non-invasive angiography. These techniques can adequately rule out $\mathrm{CAD}$, but do not provide any information on the haemodynamic consequences of the coronary artery stenoses if present. Therefore, it is likely that in the near future emphasis will shift to integration of functional and anatomical imaging. For this purpose, the diagnostic performance of PET-CT is currently being evaluated, which integrates non-invasive angiography with perfusion imaging (to visualise the haemodynamic consequences of the anatomic abnormalities). Although PET allows absolute quantification of perfusion, SPECT may be more practical in daily clinical routine, and it is anticipated that MSCT integrated with SPECT systems will become available in the future. Similarly, fusion of non-invasive angiography by MRI with functional imaging by MRI (assessment of perfusion and/or wall motion) can potentially provide similar information. Future studies should assess the additional clinical value of integrated anatomical and functional imaging, which may eventually result in superior detection and evaluation of patients with suspected and known CAD.

\section{Authors' affiliations}

J D Schuijf*, E E van der Wallt, J J Bax, Department of Cardiology, Leiden University Medical Center, Leiden, the Netherlands

H J Lamb, A de Roos, Department of Radiology, Leiden University Medical Center, Leiden, the Netherlands

L J Shaw, Atlanta Cardiovascular Research Institute, Atlanta, USA

W Wijns, Cardiovascular Center, Aalst, Belgium

D Poldermans, The Thorax Center, Erasmus Medical Centre, Rotterdam, the Netherlands

*Also at the Department of Radiology, Leiden University Medical Center, Leiden, and the Interuniversity Cardiology Institute of the Netherlands, Utrecht, the Netherlands

†Also at the Interuniversity Cardiology Institute of the Netherlands, Utrecht, the Netherlands

In compliance with EBAC/EACCME guidelines, all authors participating in Education in Heart have disclosed potential conflicts of interest that might cause a bias in the article

This work was financially supported by The Netherlands Heart Foundation, grant number 2002B105.

\section{REFERENCES}

1 Nesto RW, Kowalchuk GJ. The ischemic cascade: temporal sequence of hemodynamic, electrocardiographic and symptomatic expressions of ischemia. Am J Cardiol 1987;59:23C-30C.

Landmark paper describing the ischaemic cascade.

2 Picano E, Mathias W Jr, Pingitore A, et al. Safety and tolerability of dobutamine-atropine stress echocardiography: a prospective, multicentre 
study. Echo dobutamine international cooperative study group. Lancet 1994;344:1190-92

3 Secknus MA, Marwick TH. Evolution of dobutamine echocardiography protocols and indications: safety and side effects in 3,011 studies over 5 years. J Am Coll Cardiol 1997;29:1234-40.

4 Cerqueira MD, Weissman NJ, Dilsizian V, et al. Standardized myocardia segmentation and nomenclature for tomographic imaging of the heart: a statement for healthcare professionals from the cardiac imaging committee of the council on clinical cardiology of the American Heart Association. Circulation 2002; 105:539-42.

5 Underwood SR, Anagnostopoulos C, Cerqueira M, et al. Myocardial perfusion scintigraphy: the evidence. Eur $J$ Nucl Med Mol Imaging 2004;31:261-91.

- Comprehensive review article on the current status of myocardial perfusion imaging.

6 Wahba FF, Lamb HJ, Bax JJ, et al. Assessment of regional myocardial wall motion and thickening by gated 99Tcm-tetrofosmin SPECT: a comparison with magnetic resonance imaging. Nucl Med Commun 2001;22:663-71.

7 loannidis JP, Trikalinos TA, Danias PG. Electrocardiogram-gated singlephoton emission computed tomography versus cardiac magnetic resonance imaging for the assessment of left ventricular volumes and ejection fraction: a meta-analysis. J Am Coll Cardiol 2002;39:2059-68.

8 DePuey EG, Rozanski A. Using gated technetium-99m-sestamibi SPECT to characterize fixed myocardial defects as infarct or artifact. J Nucl Med 1995:36:952-5.

9 Smanio PE, Watson DD, Segalla DL, et al. Value of gating of technetium- $99 \mathrm{~m}$ sestamibi single-photon emission computed tomographic imaging. J Am Coll Cardiol 1997;30:1687-92.

- First study highlighting the additive value of ECG-gating to SPECT imaging.

10 Bax JJ, Van der Wall EE, De Roos A, et al. In: Zaret BI, Beller GA, eds. Clinical nuclear cardiology. State of the art and future directions. Philadelphia: Mosby, 2005:535-55.

11 Elhendy A, O'Leary EL, Xie F, et al. Comparative accuracy of real-time myocardial contrast perfusion imaging and wall motion analysis during dobutamine stress echocardiography for the diagnosis of coronary artery disease. J Am Coll Cardiol 2004;44:2185-91.

12 Porter TR, Xie F, Silver M, et al. Real-time perfusion imaging with low mechanical index pulse inversion Doppler imaging. J Am Coll Cardiol 2001;37:748-53

13 Kaul S, Senior R, Dittrich H, et al. Detection of coronary artery disease with myocardial contrast echocardiography: comparison with $99 \mathrm{mTc}$-sestamibi single-photon emission computed tomography. Circulation 1997:96:785-92.

14 Jucquois I, Nihoyannopoulos $\mathrm{P}, \mathrm{D}^{\prime}$ Hondt AM, et al. Comparison of myocardial contrast echocardiography with $\mathrm{NC} 100100$ and $(99 \mathrm{~m}) \mathrm{Tc}$ sestamibi SPECT for detection of resting myocardial perfusion abnormalities in patients with previous myocardial infarction. Heart 2000;83:518-24

15 Moir S, Haluska BA, Jenkins C, et al. Incremental benefit of myocardial contrast to combined dipyridamole-exercise stress echocardiography for the assessment of coronary artery disease. Circulation 2004;1 10:1 108-13.

- Study demonstrating the additive value of contrast administration during stress echocardiography.

16 Cwajg J, Xie F, O'Leary E, et al. Detection of angiographically significant coronary artery disease with accelerated intermittent imaging after intravenous administration of ultrasound contrast material. Am Heart $J$ 2000;139:675-83

17 Heinle SK, Noblin J, Goree-Best P, et al. Assessment of myocardial perfusion by harmonic power Doppler imaging at rest and during adenosine stress: comparison with $(99 \mathrm{~m}) \mathrm{Tc}$-sestamibi SPECT imaging. Circulation 2000;102:55-60.
18 Olszowska M, Kostkiewicz M, Tracz W, et al. Assessment of myocardial perfusion in patients with coronary artery disease. Comparison of myocardial contrast echocardiography and $99 \mathrm{mTc}$ MIBI single photon emission computed tomography. Int J Cardiol 2003;90:49-55.

19 Rocchi G, Fallani F, Bracchetti G, et al. Non-invasive detection of coronary artery stenosis: a comparison among power-Doppler contrast echo, 99Tcsestamibi SPECT and echo wall-motion analysis. Coron Artery Dis 2003; 14:239-45.

20 Shimoni S, Zoghbi WA, Xie F, et al. Real-time assessment of myocardial perfusion and wall motion during bicycle and treadmill exercise echocardiography: comparison with single photon emission computed tomography. J Am Coll Cardiol 2001;37:741-7.

21 Wei K, Crouse L, Weiss J, et al. Comparison of usefulness of dipyridamole stress myocardial contrast echocardiography to technetium $-99 \mathrm{~m}$ sestamibi single-photon emission computed tomography for detection of coronary artery disease (PB127 multicenter phase 2 trial results). Am J Cardiol 2003;91:1293-8.

22 Paetsch I, Jahnke C, Wahl A, et al. Comparison of dobutamine stress magnetic resonance, adenosine stress magnetic resonance, and adenosine stress magnetic resonance perfusion. Circulation 2004;1 10:835-42.

- Excellent study on the detection of ischaemia with MRI and different pharmacological stressors.

23 Wolff SD, Schwitter J, Coulden R, et al. Myocardial first-pass perfusion magnetic resonance imaging: a multicenter dose-ranging study. Circulation 2004:110:732-7.

24 Giang TH, Nanz D, Coulden R, et al. Detection of coronary artery disease by magnetic resonance myocardial perfusion imaging with various contrast medium doses: first European multi-centre experience. Eur Heart $J$ 2004; 25: 1657-65.

25 Al Saadi N, Nagel E, Gross M, et al. Noninvasive detection of myocardial ischemia from perfusion reserve based on cardiovascular magnetic resonance. Circulation 2000;101:1379-83.

26 Al Saadi N, Nagel E, Gross M, et al. Improvement of myocardial perfusion reserve early after coronary intervention: assessment with cardiac magnetic resonance imaging. J Am Coll Cardiol 2000;36:1557-64.

27 Togni M, Balmer F, Pfiffner D, et al. Percutaneous coronary interventions in Europe 1992-2001. Eur Heart J 2004;25:1208-13.

28 Manning WJ, Li W, Edelman RR. A preliminary report comparing magnetic resonance coronary angiography with conventional angiography. N Engl J Med 1993:328:828-32.

29 Schuijf JD, Bax JJ, Shaw $\sqcup$, et al. Meta-analysis of comparative diagnostic performance of magnetic resonance imaging and multi-slice computed tomography for non-invasive coronary angiography. Am Heart J (in press).

- Meta-analysis describing the relative merits of MRI and MSCT for noninvasive coronary angiography.

30 Moshage WE, Achenbach S, Seese B, et al. Coronary artery stenoses: threedimensional imaging with electrocardiographically triggered, contrast agentenhanced, electron-beam CT. Radiology 1995; 196:707-14

31 Budoff MJ, Achenbach S, Duerinckx A. Clinical utility of computed tomography and magnetic resonance techniques for noninvasive coronary angiography. J Am Coll Cardiol 2003;42:1867-78.

- Review on the current status of non-invasive coronary angiography.

32 Raggi P, Shaw $\sqcup$, Berman DS, et al. Prognostic value of coronary artery calcium screening in subjects with and without diabetes. J Am Coll Cardiol 2004;43:1663-9.

33 Berman DS, Wong ND, Gransar H, et al. Relationship between stress-induced myocardial ischemia and atherosclerosis measured by coronary calcium tomography. J Am Coll Cardiol 2004;44:923-30. 